9 - ORIGINAL ARTICLE

EXPERIMENTAL NEUROLOGY

\title{
Morphological and immunohistochemical analysis of apoptosis in the cerebellum of rats subjected to focal cerebral ischemia with or without alcoholism model ${ }^{1}$
}

\author{
Camila Albuquerque Melo de Carvalho ${ }^{\mathrm{I}}$, Daniela Pretti da Cunha Tirapelli ${ }^{\mathrm{II}}$, Andressa Romualdo Rodrigues ${ }^{\mathrm{III}}$, Fermino Sanches \\ Lizarte Neto $^{\mathrm{IV}}$, Paulo Cézar Novais ${ }^{\mathrm{V}}$, Jairo Pinheiro Silva ${ }^{\mathrm{III}}$, Carlos Gilberto Carlotti Júnior ${ }^{\mathrm{VI}}$, Benedicto Oscar Colli ${ }^{\mathrm{VI}}$, \\ Luís Fernando Tirapelli ${ }^{\mathrm{VII}}$
}

DOI: http://dx.doi.org/10.1590/S0102-865020160090000009

${ }^{1}$ Assistant Professor, Institute of Biological and Health Sciences, Universidade Federal de Alagoas (UFAL). Fellow PhD degree, Clinical Surgery Postgraduate Program, Department of Surgery and Anatomy, School of Medicine of Ribeirao Preto, Universidade de São Paulo (USP), Ribeirao PretoSP, Brazil. Acquisition and interpretation of data, manuscript writing.

IIAssociate Professor, Department of Surgery and Anatomy, Surgical Clinic Program, School of Medicine of Ribeirao Preto, USP, Brazil. Intellectual, conception and design of the study; manuscript writing.

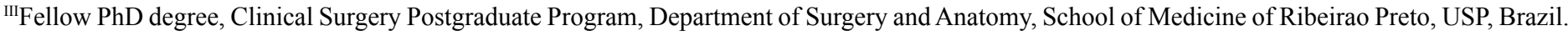
Technical procedures, manuscript writing.

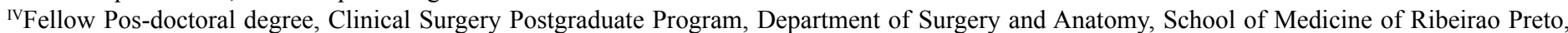
USP, Brazil.Technical procedures, manuscript writing.

vAssistant Professor, Department of Health Sciences, Universidade de Marília (UNIMAR). Fellow Pos-doctoral degree, Clinical Surgery Postgraduate Program, Department of Surgery and Anatomy, School of Medicine of Ribeirao Preto, USP, Brazil. Technical procedures, manuscript writing.

${ }^{\mathrm{V}}$ Full Professor, Division of Neurosurgery, Department of Surgery and Anatomy, School of Medicine of Ribeirao Preto, USP, Brazil. Scientific and intellectual content of the study, manuscript writing .

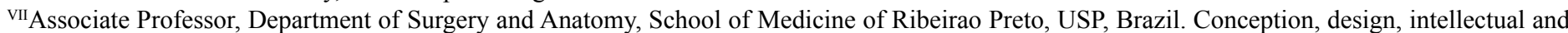
scientific content of the study; manuscript writing, critical revision; supervision of all phases of the study.

\footnotetext{
ABSTRACT

PURPOSE: To evaluated histopathological changes, morphometric and expression of proteins CASPASE-3, BCL-2 and XIAP related to apoptosis in the cerebellum after induction of temporary focal cerebral ischemia followed by reperfusion, with or without a model of chronic alcoholism.

METHODS: Fifty Wistar rats were used and divided into: control group (C), sham group (S), ischemic group (I), alcoholic group (A), and ischemic and alcoholic group (IA). The cerebellum samples collected were stained for histopathological and morphometric analysis and immunohistochemistry study.

RESULTS: Histopathological changes were observed a greater degree in animals in groups A and IA. The morphometric study showed no difference in the amount of cells in the granular layer of the cerebellum between the groups. The expression of CASPASE-3 was higher than BCL-2 and XIAP in the groups A and IA.
}

CONCLUSION: We observed correlation between histopathological changes and the occurrence of apoptosis in cerebellar cortex.

Key words: Ischemia. Alcoholism. Cerebellum. Apoptosis. Morphology. Rats. 


\section{Introduction}

The mortality and morbidity caused by cerebral ischemia worldwide are high ${ }^{1,2}$. The wide diversity in its anatomical location, etiology, and clinical manifestations become the cerebral ischemia a disease that has difficult therapeutic control ${ }^{3,4}$.

Several structural and biochemical changes in animals subjected to cerebral ischemia can be observed in areas distant from the ischemic focus ${ }^{5}$. Studies have shown that the cerebellum is one of these areas. There are reports in the literature demonstrating that apoptosis is one of the mechanisms triggered in the cerebellum during focal cerebral ischemia ${ }^{6}$. Associated with cerebral ischemia, many harmful agents are studied. One of these agents is alcoholism, considered one of the most serious public health problems in many countries, both by complications in somatic and psychic plane, as by the deep social repercussions ${ }^{7}$. Chronic alcohol consumption exacerbates transient ischemic brain injury and this increase in ischemic brain damage during alcohol consumption may be related to deficiency in cerebral vasoreactivity ${ }^{8}$. According to studies of psychology, neuropsychology, neuroimaging and neuropathology, the frontal lobe, the limbic system and the cerebellum are particularly vulnerable to injury and disorders related to excessive alcohol use ${ }^{9}$.

The cells in the nervous tissue do not behave in a uniform manner when they are affected by ischemia. Endothelial cells are more resistant than the glial cells and these in turn are more resistant than neurons. Even comparing only neurons, some of them exhibit a greater susceptibility to ischemia than others, for example, hippocampal neurons are highly susceptible to ischemia ${ }^{10}$. Studies have been conducted in an attempt to correlate cerebral and cerebellar ischemia.

The structural and biochemical changes in brain after ischemia animals may be far from ischemic focus. Therefore, edema compressed cells in the contralateral hemisphere. Depolarization of cells caused by intracellular calcium causes the cortical spreading depression. The cortical spreading depression waves cause metabolic stress and can lead to the induction of various inflammatory mediators, growth factors and heat shock proteins that do not exactly coincide with the infarcted area ${ }^{5}$.

The decreased blood flow in only one region of the central nervous system compromises cell integrity and determines the focal ischemia. This process can lead to apoptosis, necrosis or both ${ }^{11,12}$. A family of proteins B cell lymphoma 2 (BCL- 2) is a necessary and essential component in the regulation of cell death by apoptosis. The proteins of this family can induce apoptosis: $\mathrm{x}$ associated protein BCL -2 (BAX), killer/ antagonist of BCL -2 (BAK) or may inhibit apoptosis: BCL-2 and as long BCLX (BCL -XL). Another important mechanism of apoptosis is mediated by the protein XIAP, a potent caspase inhibitor, with different effects of BCL-2. It has action at a distal point of death pathway and its activity has been shown to be fundamental in neuronal degeneration after ischemia and other injuries ${ }^{13}$.

Therefore, our goal was to evaluate the possible histopathological changes and analyze the expression of proteins related to the mechanism of apoptosis through the expression of caspase- 3, BCL -2 and XIAP protein in the cerebellum of adult male rats submitted to of transient focal cerebral ischemia, associated or not with chronic alcoholism.

\section{Methods}

The experiments were carried out according to the Ethical Principles for Experimental Animals (COBAO) and the study was approved by the Animal Experimentation Committee (CETEA) of the Ribeirão Preto Medical School, Universidade de São Paulo (USP).

Fifty young adult male rats of the Wistar strain (Rattus norvegicus) weighing approximately 200 grams (6-7 weeks) at the beginning of the experiment and 280-310 grams at the end of the experiment were used. The animals were randomly divided into five experimental groups with 10 animals each: control (C), animals euthanized without submitting to the surgical procedure; sham (S), animals submitted to complete simulation of the surgical procedure but without ischemia and then euthanized; ischemic (I), animals submitted to focal ischemia for 90 minutes followed by reperfusion for 48 hours, and then euthanized; alcoholic (A), animals that received $20 \%$ ethanol for four week sand they were euthanized, and, ischemic and alcoholic (IA): animals subjected to the same treatment from group A and they were submitted tofocal cerebral ischemia for 90 minutes followed by reperfusion for 48 hours. Weekly measurements of the weight of the animals were heldin the differentstudy groups.

The preparation of the alcoholic groups was conditioned to a brief period of gradual adaptation to the consumption of ethanol, which consisted in supplying ethanol diluted in water with its concentration amended gradually in increasing doses weekly of $5 \%, 10 \%$ and $20 \%$; the experimental phase started at the beginning of third week of treatment.

\section{Induction of cerebral ischemia}

All animals were partially anesthetized by halothane inhalation and intubated with an orotracheal cannula. Twice during the ischemic period, arterial blood samples were collected for the determination of glycemia, $\mathrm{paCO}_{2}, \mathrm{paO}_{2}$ and $\mathrm{pH}$. MCA occlusion was carried out through the external carotid artery (ECA) which 
was ligated cranially and sectioned for the retrograde introduction of a $2.5 \mathrm{~cm}$ long obstructive 4-0 mononylon suture with one end thickened with silicone over an extension of $5 \mathrm{~mm}$. The suture was introduced until to reach the common carotid artery (CCA) and then cranially progressed through the internal carotid artery (ICA) until to reach and to obstruct the MCA.

After the period of ischemia, we proceeded to the removal of the obstructing thread, placing temporary clamps on the CCA and on the ICAto prevent blood flow reflux and the the proximal stumpof the ECA was definitively linked (cotton yarn 4.0-Ethicon). The skin and the subcutaneous tissue were closed in animals of the groups I and IA. The animals of these groups after reperfusion 48 hours were euthanized. For the animals of group A, euthanasia was performed after the treatment period with $20 \%$ ethanol.

After euthanasia, the removal of the brain was carried out which was placed in a steel mold (Matrix model - ASI - Instruments - CBM - 2000C U.S.A.) used for performing coronal sections. For the study by light microscopy (LM) and immunohistochemistry (IH), we used a coronal section ( $2 \mathrm{~mm}$ ) whose section is performed through the middle of the cerebellum region. After obtaining the coronal section was initiated manual immersion fixative solution of $4 \%$ paraformaldehyde in $0.1 \mathrm{M}$ phosphate buffer, $\mathrm{pH} 7.3$ at room temperature to the study by the LM and $\mathrm{IH}$.

From coronal sections were obtained $3 \mu \mathrm{m}$ thick sections and stained (Trichrome Masson's stain) for histopathological and morphometric analysis and prepared for immunohistochemistry for protein expression of Caspase- 3, BCL- 2 and XIAP. Histopathological changes and immunohistochemistry study were evaluated in the neurons of the left cerebellar cortex and not in glial cells.

For morphometric analysis was carried out by counting the number of cells of the granular layer of the cerebellum left hemisphere at x400 magnification.

For analysis of protein expression were selected two fields at 400x magnification, where there was a greater concentration of positive cells or marked (areas of "hot spots") for each protein under study. From the count of the total number of positive and negative cells, it was calculated the percentage of positive cells. The protein expression analysis was performed in the cerebellar neurons for counting performing a comprehensive evaluation of molecular layer, Purkinje cells and granular cells. The samples were submitted to immunohistochemical analysis by the method of avidin-biotin-peroxidase (Novostain Super ABC Kit - Universal, NCL-ABCu, Novocastra Laboratories Ltd, Newcastle upon Tyne, United Kingdom) - (universal Kit mach 4 BIOCARE), and incubation cerebellar tissue for 24 hours in primary antibodies related to the mechanism of apoptosis: Caspase-3 (pro-apoptotic -
229 CP-B / rabbit polyclonal / Biocare Medical) diluted 1/300 in bovine serum albumine - $1.5 \%$ BSA, BCL2 (anti- apoptotic - C-2 / sc-7382 / mouse monoclonal IgG1 / Santa Cruz) diluted at 1/200 in bovine serum albumine - $1.5 \%$ BSA and XIAP (anti-apoptotic - H-202 / sc-11426 / rabbit polyclonal IgG / Santa Cruz) diluted at $1 / 300$ in bovine serum albumine $-1.5 \%$ BSA.

The reactions with positive control for caspase-3 were performed using rat tonsil, for BCL2 rat skin and for XIAP rat thymus (recommended). The negative control reactions were performed with the same tissue under study: cerebellum without the use of primary antibody. These samples were kept in blocking solution (normal horse serum).

The samples were incubated with the secondary antibody kit MACH 4 Universal HRP-Polymer (M4BD534, Biocare Medical) and then with avidin-biotin-peroxidase kit same (1/200 in PBS). Finally, reactions were developed with diaminobenzidine solution (3, 3 '- Diaminobenzidine tetrahydrochloride, SigmaAldrich, Saint Louis, Missouri, USA) and the sections were counterstained with Harris hematoxylin. The slides were analyzed using the Zeiss microscope Axioskop 2 plus model at $\mathrm{x} 400$ magnification. The images of the fields used for quantification of proteins were recorded by the camera (Axio Cam $\mathrm{Hrc}^{\circledR}$ ) attached to the microscope, and subsequently filed the Axio Vision $4.6^{\circledR}$ program.

Data concerning the mean protein expression and the morphometric analysis in the many groups were analyzed statistically by Kruskal-Wallis test followed by the Bonferroni post-test using the GraphPad Prism software, version 6.00 for Windows, (GraphPad Software, San Diego, CA, USA). The level of significance was set at $\mathrm{p}<0.05$ for two-tailed tests.

\section{Results}

\section{Histopathological analysis}

In some animals of group IA, it was observed the presence of some pockets of interstitial edema mainly located in the transition between the granular and molecular layers in the presence of Purkinje cells, presenting some aspects of nuclear condensation, pyknotic nuclei as well, suggesting the same finding also to cells of the granular layer Golgi (Figures 1 and 3A).

Leukocyte infiltration or inflammation as well as the presence of hemorrhagic foci were not observed, in animals of all groups (Figure 2). A common finding in animals of groups A, I, and particularly IA, is the presence of granule cells with pyknotic nuclei dispersed throughout the granular layer, especially in the outside closer to the Purkinje cells. 
The morphometric study showed no difference in the amount of cells in the granular layer of the cerebellum between all experimental groups $(\mathrm{p}=0.3499$, Kruskal-Wallis test).

The expression of caspase- 3 (Figures $3 \mathrm{~B}$ and 4) was higher than BCL -2 and XIAP expressions in the groups A and IA, especially near the transition between the granular and molecular layer. The expression of caspase- 3 and BCL-2 protein was higher in the granular and molecular layers (Figures 3B to 9) of the cerebellar cortex in the ischemic, alcoholic, and alcoholic + isquemic groups.

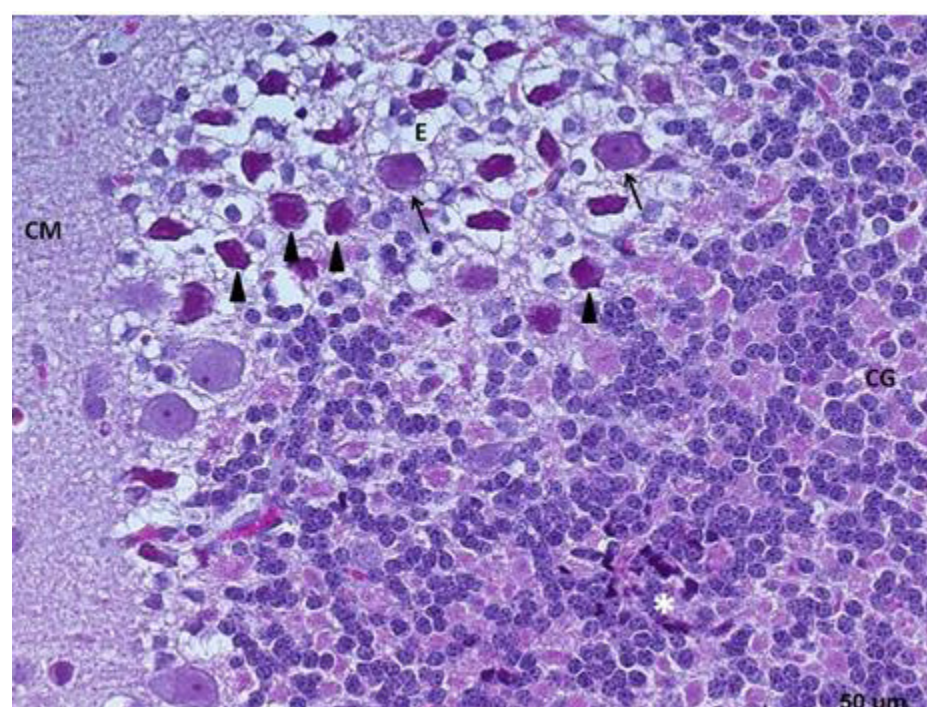

FIGURE 1 - Photomicrograph of the cortex of left cerebellar hemisphere of one animal of Group IA in the transition between the molecular layer (ML) and granular layer (CG). Note interstitial edema (E), cells with pyknotic nuclei ( ) in the upper region, as well as two Purkinje cells with nuclear and cytoplasmic condensation $(\rightarrow)$. Downwards, darker pyknosis is seen in a few cells in the granular layer (*). Masson's Trichrome; $x 400$.

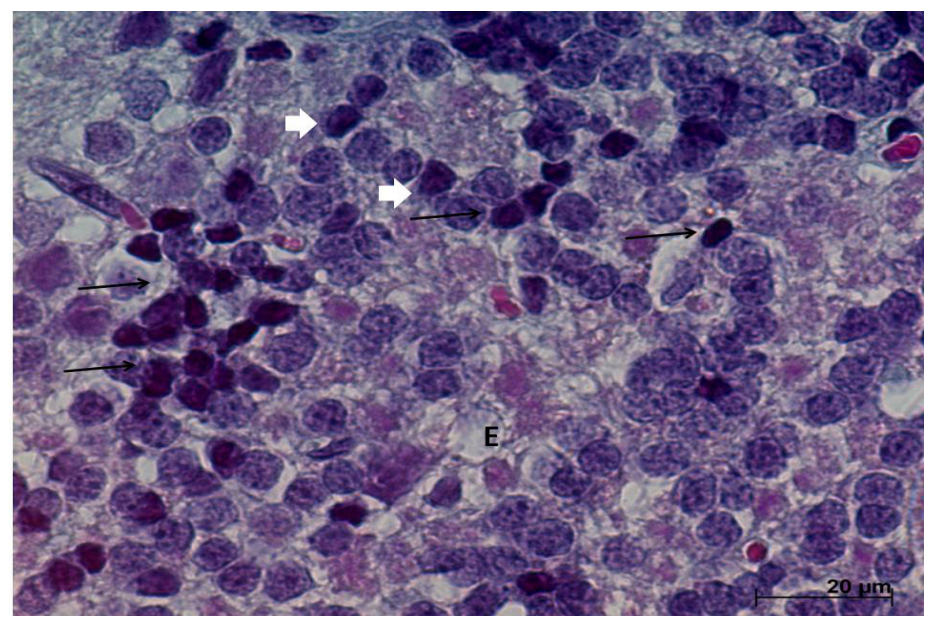

FIGURE 2 - Photomicrograph of the cortex of the left cerebellar hemisphere of one animal of Group IA. Note granular detail with many granular layer cells of normal aspect, with circular containing nuclei, surrounded by granule cells with condensed karyopyknosis $(\rightarrow)$. Featured (white arrows) in two granular cell nuclear condensation process. Edema in the central region (E), Masson's Trichrome; x1000.

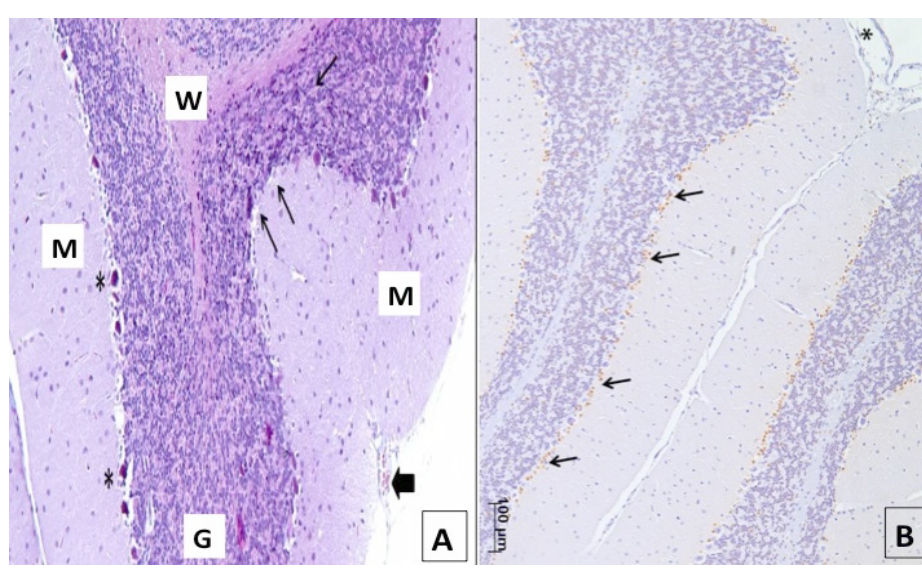

FIGURE 3 - A. Photomicrograph of the cortex of two sheets of the left cerebellar hemisphere of an animal group I + A. The thinner arrows indicate the region of the granular layer $(\mathbf{G})$ with the presence of granule cells darker staining pyknotic nuclei, especially at the transition to the molecular layer (M). The thick arrow shows a blood vessel of the pia mater, the outer covering of the molecular layer. Purkinje cells $\left(^{*}\right)$; white matter (W). Masson; x100. B. Photomicrograph overview of two sheets cerebellar left cerebellar hemisphere with positive nuclear staining for caspase- 3 mainly in the transition region between molecular and granular layers (arrows) of an animal of group I+A. Pia mater (*); x100.

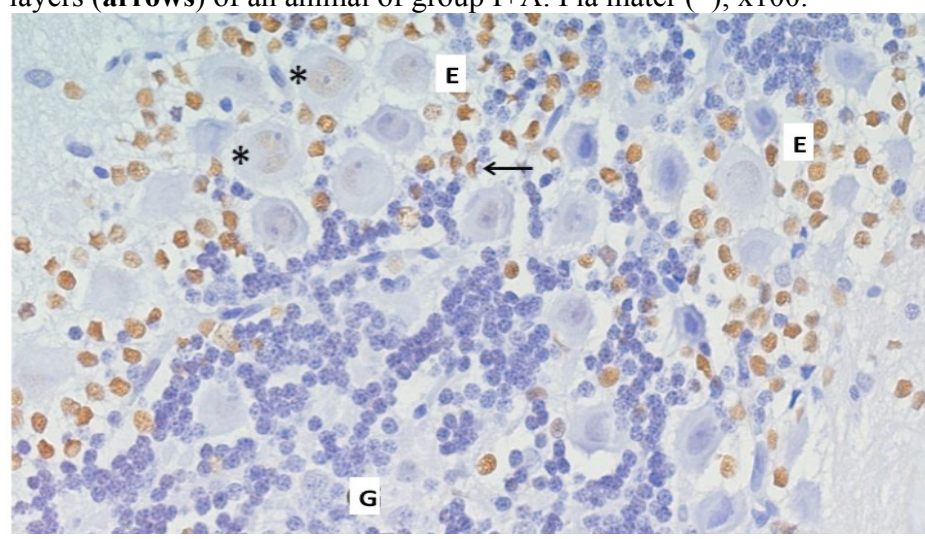

FIGURE 4 - Photomicrograph the left hemisphere cerebellar cortex with positive nuclear staining caspase 3. Detail showing the transition region between the molecular and granular layers $(\mathbf{G})$ of an animal of group I + A, with slight edema $(\mathbf{E})$ and focal positive staining around the Purkinje cells (arrowheads) and low positive staining intensity in the core of two Purkinje cells (*). Observe positive staining for some granular cells; 400 .

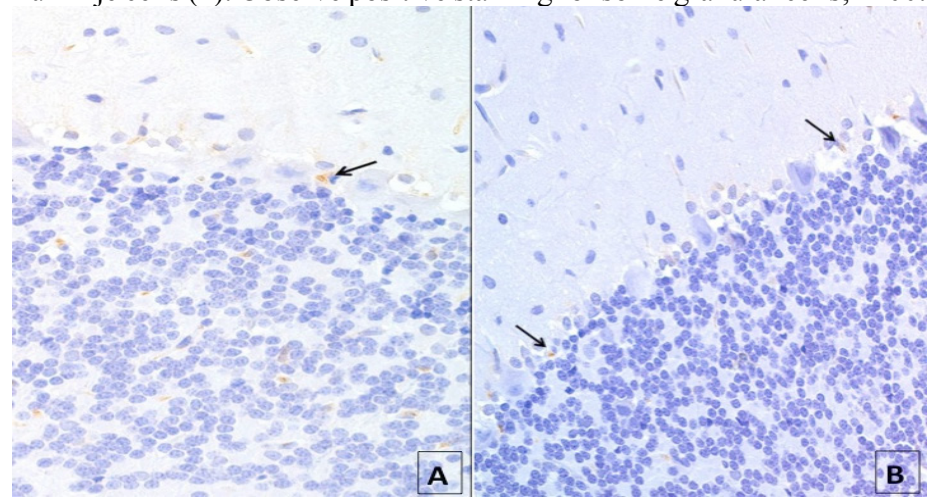

FIGURE 5 - Photomicrograph of the left cerebellar hemisphere of a Group I animal with low expression (arrows) for BCL2 (A) in the transition region between molecular and granular layers and also in the granular layer; and a group I animal with few cells with positive staining for XIAP (arrows) (B) in the transition region between the molecular and granular layers. $\mathrm{X} 400$. 
CASPASE 3 granular layer

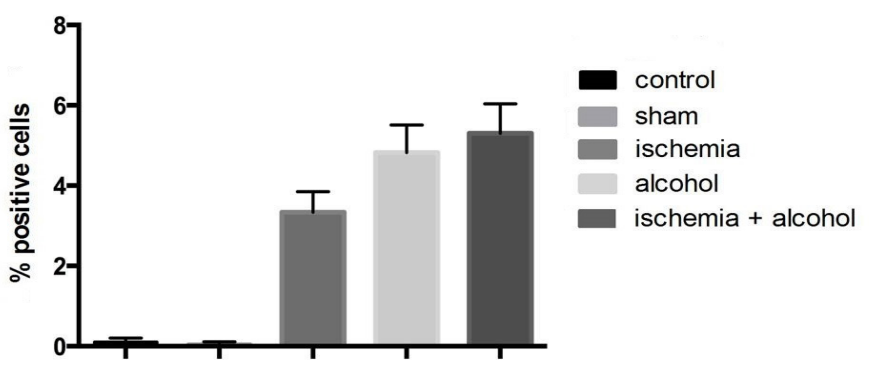

FIGURE 6 - Mean ( \pm standard deviation) of the protein expression of CASPASE-3 in the granular layer of the cerebellum in the animals of all experimental groups $\mathrm{p}<0.0001$, Kruskal-Wallis test. There was a statistically significant difference between groups: CxA, CxIA, SxA $(\mathrm{p}<0.001)$ and SxIA $(\mathrm{p}<0.001) * *$, Dunns post-test. ${ }^{* *}$ Control $(C)$, Sham (S), Ischemia (I), Alcohol (A), Ischemia + Alcohol (IA).

\section{CASPASE 3 molecular layer}

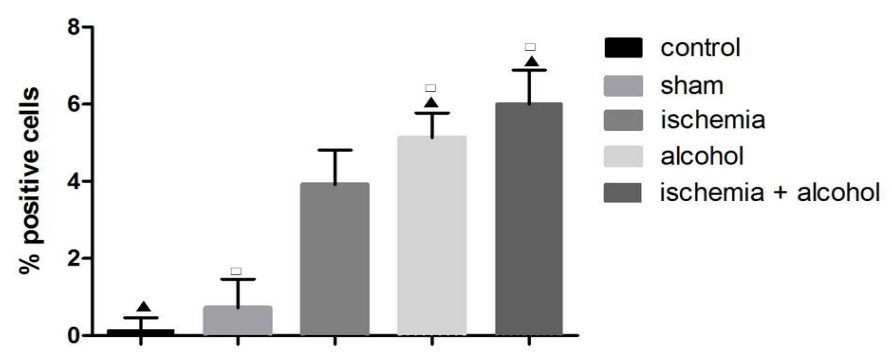

FIGURE 7 - Mean ( \pm standard deviation) of the protein expression of CASPASE-3 in the molecular layer of the cerebellum in animals of all experimental groups $\mathrm{p}<0.0001$, Kruskal-Wallis test. There was a statistically significant difference between groups: C x IA ( $<<0.0001)$; C x A, S x IA $(\mathrm{p}<0.001)$ and S x A $(\mathrm{p}<0.05)^{* *}$, Dunn's post-test. **Control (C), Sham (S), Ischemia (I), Alcohol (A), Ischemia + Alcohol (IA).

BCL2 granular layer

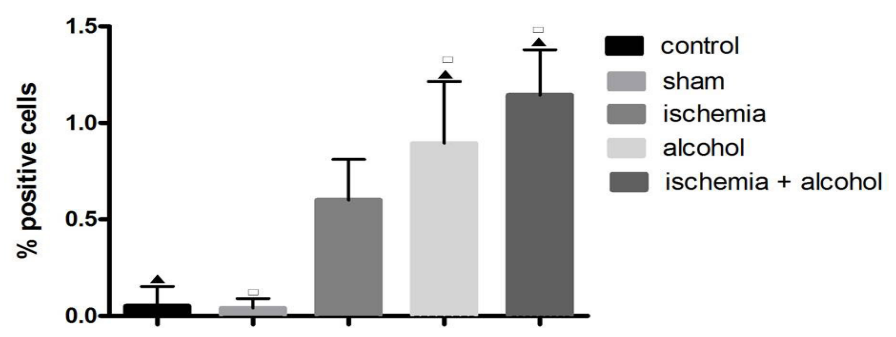

FIGURE 8 - Mean ( \pm standart deviation) of the protein expression of BCL2 in the granular layer of the cerebellum in animals of all experimental groups $\mathrm{p}<0.0001$, Kruskal-Wallis test. There was a statistically significant difference between groups: CxIA, SxIA $(p<0.0001)$; CxA $(p<0.001)$ and SxA $(\mathrm{p}<0.05)^{* *}$, Dunn's post-test. **Control (C), Sham (S), Ischemia (I), Alcohol (A), Ischemia + Alcohol (IA).

\section{BCL2 molecular layer}

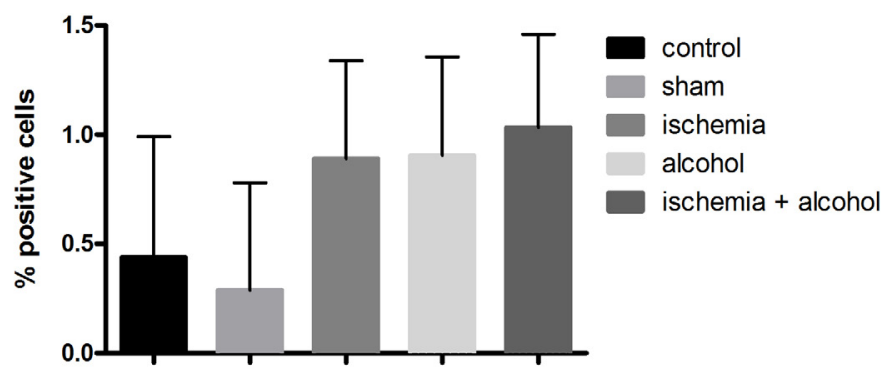

FIGURE 9 - Representation of the mean ( \pm standard deviation) of the protein expression of BCL-2 in the molecular layer of the cerebellum in animals of all experimental groups ( $\mathrm{p}=0.0370$, Kruskal-Wallis test).

\section{The results showed that the expression of XIAP protein}

was low in the granular layer of the cerebellum in the ischemic, alcoholic, and ischemic + alcoholic groups (Figures 10 and 11). In regions where the histopathological changes were more intense (boundary between the granular and molecular layers), the expression of caspase- 3 was higher. These data suggest that reduced cell viability.

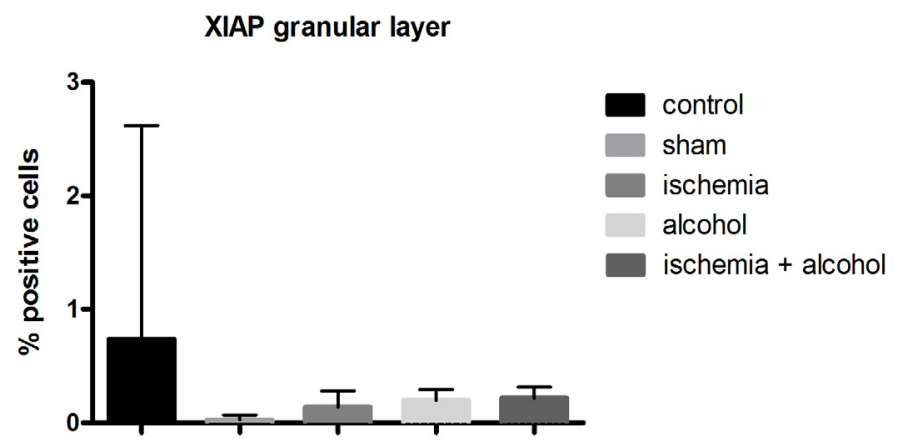

FIGURE 10 - Mean ( \pm standard deviation) of XIAP protein expression in the granular layer between the groups $(\mathrm{p}<0.0057$, Kruskal-Wallis, and $\mathrm{C}$ x I C x IA ( $\mathrm{p}<0.05)$, Dunn's post-test.

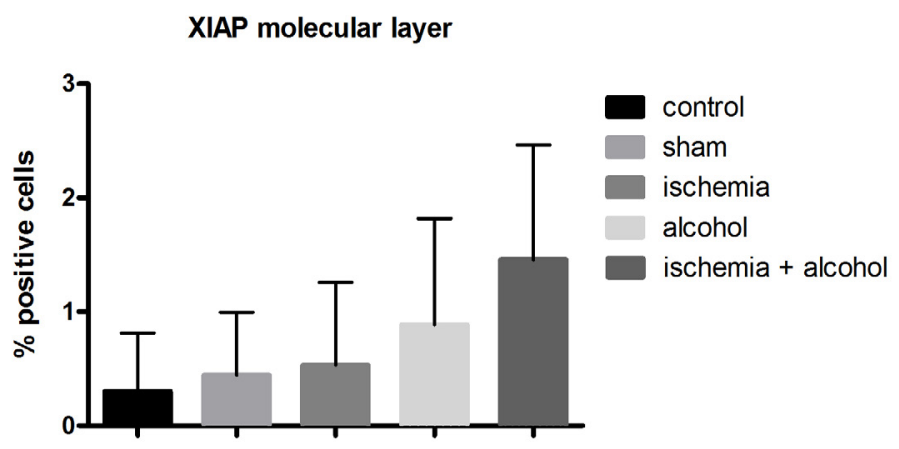

FIGURE 11 - Representation of the mean ( \pm standard deviation) of XIAP protein expression in the molecular layer between the groups $(p=0.1622$, Kruskal-Wallis). 


\section{Discussion}

The focal or global cerebral ischemias are very common diseases in the human population. Because of the great number of variables involved in the ischemic process, many animal models are well established for the study of ischemia ${ }^{14}$. The global ischemia causes lesions in some brain areas that are selectively vulnerable as neurons of the striatum and and the Purkinje cells of the cerebellum ${ }^{15}$, and instead damage caused by focal cerebral ischemia are also observed in regions other than the ischemic focus ${ }^{16}$. Alcoholism, is classified as one of the most serious public health problems in many countries, and it is known that the effects of alcohol are known to be severe in brain tissue, especially in the cerebellum. Based on these premises, this study aimed to provide information on the possible contribution of apoptosis to neurodegeneration of the cerebellum of animals subjected to focal ischemia from occlusion of middle cerebral artery and to the effects of chronic alcoholism.

Peng et al. ${ }^{16}$ conducted a study with focal cerebral ischemia model by permanent occlusion of the right common carotid artery in rats with seven days of life. Samples of cerebellar tissue and the brainstem were analyzed by 2-deoxyuridine 5-triphosphate-biotin technique (TUNEL). Positive cells to apoptosis and necrosis were presented distributed both in the cerebellum and in the brainstem. Positive cells in the cerebellum were confined to external germinal layer, although positive cells randomly distributed in the granular layer were observed. Apoptotic and necrotic positive staining were clearly distinguished both in the germline as the outer layer of the granular layer. Some cells showed clearly necrotic morphology with edema and oversized, while others were smaller and with apoptotic morphology and the presence of apoptotic bodies. In the outside layer of the cerebellum, the number of apoptotic cells was significantly increased when compared to the necrotic cells. However, in the granular layer, the number of apoptotic and necrotic cells were similar. These findings indicate that the mechanism of apoptosis was triggered in the cerebellum and brainstem regions that are distant from the primary ischemic region. Therefore, the authors concluded that although the cerebellum and brainstem are outside the vascular supply of the ligated artery, these regions suffered the actions of neurodegeneration ${ }^{16}$.

In our study, we observed in some animals of group IA (Ischemic + alcoholic), the presence of some pockets of interstitial edema, mainly located in the transition between the granular and molecular layers and the presence of Purkinje cells presenting some aspects of nuclear condensation and pyknotic nuclei as well the same finding for Golgi cells of the granular layer. The edema was more intense in some animals of group A and IA. Therefore, our results also showed lesions in the cerebellar tissue distant from the ischemic focus region and associated with alcoholism.

The BCL-2 family possesses both pro- apoptotic proteins and anti - apoptotic proteins and activation of these proteins that determine cell death or not. In the cerebellum, acute exposure to ethanol increases the expression of pro-apoptotic proteins, such as BAX, BAD, BCL-xs and decrease the expression of anti -apoptotic proteins such as Bcl- 2 protein. Increased BCL2 expression protects Purkinje cells, but does not have the same effect on granule cells. Luo ${ }^{17}$ also describes the rat cerebellum is particularly vulnerable to ethanol during the first 10 days after birth and that this exposure during this period of time causes the death of Purkinjeand granule cells. However, granule cells may have greater sensitivity to ethanol. Some researchers suggest that the loss of granule cells can occur as a secondary slow reaction to the loss of Purkinje cells.

Jie et al..$^{18 \text { from a model of ischemia by occlusion }}$ of the middle cerebral artery in rats observed some changes of cerebelar cortex. The results indicated apoptosis after immunohistochemistry analyses showed by increased expression of Caspase 3 in neural cells of cerebellar cortex even as mechanism of secundary injury of main infarction.

Our results showed increased expression of BCL - 2 protein in both groups subjected to cerebral ischemia as chronic alcoholism, alone or combined. According to $\mathrm{Luo}^{17}$, with the increase in its expression, the protective action (anti-apoptotic) of the BCL-2 protein, occurs in the Purkinje cells, but does not have the same effect in the granular layer. However, mostly it observed positive for CASPASE -3 in Purkinje cells. Our study assessed only the granular and molecular layers, with low expression of BCL-2, but was slightly higher in the granular layer of the animals in group IA compared to the molecular layer. In the groups A and I the expression of BCL-2 was low and it was similar in both layers (granular and molecular). Compared to the expression of caspase3 , the expression of BCL-2 was lower, indicating reduced antiapoptotic effect, especially in the regions of greatest labeling for caspase -3 .

A common morphological finding in animals in groups A, I, and especially in IA was the presence of granule cells with pyknotic nuclei found dispersed throughout the granular layer,however, mainly found in the outermost region of this layer that is closer to the Purkinje cells located. Hara et al. ${ }^{19}$ reported the findings in the cerebellum at necropsy in two patients with a history of a single episode of transient global cerebral ischemia. In 
both cases, no apparent cell death was found in the granular layer sections stained with hematoxylin eosin (HE). In sections with specific staining for DNA, in situ DNA fragmentation was observed in approximately one third of the granule cells of the cerebellar cortex, with the morphology of itsnuclei relatively preserved. In the Purkinje cells, no DNA fragmentation was identified in the nuclei, as well as death was not seen by HE staining. This event suggests that even in instances of mild transient global cerebral ischemia, nuclear fragmentation occurred exclusively in a particular group of cells in the granular cell layer, not affecting the Purkinje cells.

The thinning of the granular cell layer is usually seen associated with changes in Purkinje cells after several weeks of global cerebral ischemia. The decrease of the granule cell layer suggests that some groups of these cells are more vulnerable to ischemia thanothers. Possibly, the mechanism of apoptosis is responsible for the atrophy of this layer after ischemia. Hara et al. ${ }^{19}$ reported that Purkinje cells are more vulnerable to ischemia cells of the granular layer. However, in this study, the findings suggest that there is a difference of vulnerability within the group of granule cells. The type of granule cells would be more vulnerable to Purkinje cell. However, the range of the vulnerability of each cell type in the granular layer of the cerebellum is not yet understood.

In this study we observed decreased expression of anti - apoptotic protein XIAP in the granular layer in the groups submitted to cerebral ischemia and alcoholism, alone or combined, when compared with the control group. However, in the molecular layer, the XIAP protein expression was not different from the control groups. Based on the pattern of expression of XIAP protein in the granular and molecular layers our results corroborate the findings of Hara et al. ${ }^{19}$ regarding differences in the vulnerability of cell types. When compared to the expression of BCL-2, XIAP expression was lower in the two analyzed layers.

Olney et al. ${ }^{20}$ conducted studies with seven days old C57BL/6 micetreated with ethanol. The DeOlmos and immunocytochemical staining with silver were used to demonstrate the activation of caspase- 3 . They analysis the neural tissue by light and electron microscopy at intervals of $4,8,12$, 16, 20, 24, 48 and 72 hours after the first dose of ethanol. The mice sacrificed 24 hours after ethanol administration revealed a diffuse pattern of neurodegeneration affecting various brain regions such as cortical layers of the frontal, parietal, temporal, occipital and cingulate. Increase of the activation of caspase-3 response was observed in the same population of cells that was selectively stained with DeOlmos: these findings observed about 4 hours after the first dose of ethanol and becamemore evident after 8 to 10 hours. The evaluation by microscopy performed at different intervals, demonstrated various stages of evolution of the apoptotic mechanism. The first ultrastructural changes consist in forming a spherical dough changes in chromatin and nucleoli. During this first stage, both the nuclear membrane and cytoplasmic organelles remain relatively unchanged with the exception of some mitochondria that became swollen. The steps in the medium and long term included a progressive fragmentation of the nuclear membrane, mixing between nuclear and cytoplasmic contents and gradual condensation of the cell. The earliest stage presents the evident presence of apoptotic bodies, besides not being possible, the distinction between nucleus and cytoplasm.

In our study we observed overexpression of caspase-3in the granular layer, and especially in the molecular layer in the groups submitted to cerebral ischemia and chronic alcoholism when compared to the control group. The positive staining for caspase- 3 was observed mainly in the transition between the two layers of the cerebellum and it was also been observed positive staining of Purkinje cells. These results confirm data from the literature, whereas caspase- 3 is an ordinary member of the extrinsic and intrinsic pathways of apoptosis. Thus, we conclude that the mechanism of apoptosis wasactivated in the cerebellum of animals in this study.

In experiment with seven days old mice treated with ethanol, Olney et al. ${ }^{20}$ found degenerative response induced by ethanol observed by transmission electron microscopy, and they observed the characteristics of cell death by apoptosis. Furthermore, reported that during the period of higher brain growth, different neuronal populations became sensitive at different times mechanism by which the apoptotic degeneration occurs. The authors reported that the bigger the time of exposure to blood ethanol concentrations above $200 \mathrm{mg} / \mathrm{dl}$, degenerative responses became progressively more severe and more widespread. Yet, according to the authors, ethanol acts on the different cell groups causing apoptotic neuronal degeneration and variety of neurobehavioral disorders.

Oliveira et $a l^{21}$ examined the effect of ethanol on apoptosis Golgi, Purkinje and granule cells of the cerebellum. A group of 20 mice consumed UChA 10\% ethanol ( $<2 \mathrm{~g}$ ethanol $/ \mathrm{kg}$ body weight/day) and after 120 days of age, the cerebellum of these animals were collected and subjected to immunohistochemistry for terminal deoxynucleotide transferase dUTP nick end labeling (TUNEL), caspase-3, XIAP, and insulin-like growth factor-1 receptor (IGF-1R); real-time PCR (RT-PCR) to determine, XIAP and IGF-1R expression of the caspase-3 gene; and transmission electron microscopy (TEM). DNA fragmentation and an increase 
in caspase-3 and XIAP protein in Purkinje cells were identified, while the granular cells showed an increase in caspase- 3 and XIAP. There was an increase in lipid droplets in the cytoplasm reduction in electron-dense nuclei and changes in the myelin sheath in the cerebellar cortex. The other parameters evaluated did not show changes and the study concluded that the ethanolinduced apoptosis in Purkinje and granule cells of the cerebellum of adult rats UChA.

Martinez et al. ${ }^{22}$ using the same strain of animals (UChA) evaluated the ultrastructural alterations and apoptosis in the cerebellar white matter in a group of animals that consumed ethanol $10 \%$ for 120 days compared to the control group. Severe ultrastructural changes were observed in UChA group such as increase in the interaxonal spaces, disruption of the myelin sheath and disorganization and deformation of its componentes. The immunohistochemistry study for caspase- 3 showed higher expression in animals treated with ethanol when compared to the control group and low expression of anti-apoptotic protein XIAP both in animals treated with ethanol as the control group. Therefore, the authors showed that use of ethanol leads to significant ultrastructural alterations in the glial cells of the cerebellar white matter, as well as programmed cell death of these cells in these animals ${ }^{22}$.

This work allowed us to observe the activation of the mechanism of apoptosis in the cerebellum of animals subjected to both cerebral ischemia and to chronic alcoholism. However, more studies are needed to understand the role of other anti and proapoptotic proteins.

\section{Conclusions}

Histopathological changes were observed greater degree in animals in groups A and IA, the three layers of the cerebellum. The morphometric study showed no difference in the amount of cells in the granular layer of the cerebellum between the groups. The expression of caspase- 3 was higher than Bcl -2 and XIAP expression, increased with groups A and IA, especially near the transition region between the granular and molecular layer.

Although it has been observed correlation of morphological changes and increased protein expression of Caspase 3, indicating apoptosis, especially in animals group IA; the comparison between the groups for each individual analysis (morphological and immunohistochemical) found very similar results. Thus, we can conclude that from the experimental model used for cerebral ischemia induction, the lesions secondary to ischemic region of focus, were not intense in the cerebellar tissue.

\section{References}

1. Conway EM, Zwerts F, Van Eygen V, DeVriese A, Nagai N, Luo W, Collen D. Survivin-dependent angiogenesis in ischemic brain: molecular mechanisms of hypoxia-induced up-regulation. Am J Pathol. 2003;163(3):935-46. PMID: 12937134.

2. Shalak L, Perlman JM. Hypoxic-ischemic brain injury in the term infant-current concepts. Early Hum Dev. 2004;80(2):125-41. PMID: 15500993.

3. Bratina P, Greenberg L, Pasteur W, Grotta JC. Current emergency department management of stroke in Houston, Texas. Stroke. 1995;26(3):409-14. PMID: 7886715.

4. Carlotti CG, Colli BO, Kazuo JY. Evaluation of brain ischemia by mitochondrial respiration: experimental model. Arq Neuropsiquiatr. 2001;59(2-B):365-71. PMID: 11460181

5. Leker RR, Shohami E. Cerebral ischemia and trauma-different etiologies yet similar mechanisms: neuroprotective opportunities. Brain Res Brain Res Rev. 2002;39(1):55-73. PMID: 12086708.

6. Liu F, Lo CF, Ning X, Kajkowski EM, Jin M, Chiriac C, Gonzales C, Naureckiene S, Lock YW, Pong K, Zaleska MM, Jacobsen JS, Silverman S, Ozenberger BA. Expression of NALP1 in cerebellar granule neurons stimulates apoptosis. Cell Signal. 2004;16(9):101321. PMID: 15212762.

7. Gilmore W, Chikritzhs T, Stockwell T, Jernigan D, Naimi T, Gilmore I. Alcohol: taking a population perspective. Nat Rev Gastroenterol Hepatol. 2016;13(7):426-34. PMID: 27188823.

8. Sun M, Zhao Y, Gu Y, Xu C. Neuroprotective actions of aminoguanidine involve reduced the activation of calpain and caspase-3 in a rat model of stroke. Neurochem Int. 2010;56(4):63441. PMID: 20116408.

9. Oscar-Berman M, Marinković K. Alcohol: effects on neurobehavioral functions and the brain. Neuropsychol Rev. 2007;17(3):239-57. PMID: 17874302.

10. Church J, Zeman S, Lodge D. The neuroprotective action of ketamine and MK-801 after transient cerebral ischemia in rats. Anesthesiology. 1988;69(5):702-9. PMID: 2847595.

11. Colli BO, Tirapelli DP, Carlotti CG, Lopes LaS, Tirapelli LF. Biochemical evaluation of focal non-reperfusion cerebral ischemia by middle cerebral artery occlusion in rats. Arq Neuropsiquiatr. 2008;66(3B):725-30. PMID: 18949271.

12. del Zoppo GJ. Virchow's triad: the vascular basis of cerebral injury. Rev Neurol Dis. 2008;5 Suppl 1:S12-21. PMID: 18645567.

13. Clem RJ, Duckett CS. The iap genes: unique arbitrators of cell death. Trends Cell Biol. 1997;7(9):337-9. PMID: 17708972.

14. Pulsinelli WA, Buchan AM. The four-vessel occlusion rat model: method for complete occlusion of vertebral arteries and control of collateral circulation. Stroke. 1988;19(7):913-4. PMID: 3291205.

15. Nakka VP, Gusain A, Mehta SL, Raghubir R. Molecular mechanisms of apoptosis in cerebral ischemia: multiple neuroprotective opportunities. Mol Neurobiol. 2008;37(1):7-38. PMID: 18066503.

16. Peng JH, Feng Y, LeBlanc MH, Rhodes PG, Parker JC. Apoptosis and necrosis in developing cerebellum and brainstem induced after focal cerebral hypoxic-ischemic injury. Brain Res Dev Brain Res. 2005;156(1):87-92. PMID: 15862631.

17. Luo J. Mechanisms of ethanol-induced death of cerebellar granule cells. Cerebellum. 2012;11(1):145-54. PMID: 20927663.

18. Jie L, Yuqin C, Yao D, Shuai H, Yuanyuan W. Occlusion of middle cerebral artery induces apoptosis of cerebellar cortex neural cells via caspase-3 in rats. Turk Neurosurg. 2011;21(4):567-74. PMID: 22194118.

19. Hara A, Yoshimi N, Hirose Y, Ino N, Tanaka T, Mori H. DNA fragmentation in granular cells of human cerebellum following global ischemia. Brain Res. 1995;697(1-2):247-50. PMID: 8593583. 
20. Olney JW, Tenkova T, Dikranian K, Qin YQ, Labruyere J, Ikonomidou C. Ethanol-induced apoptotic neurodegeneration in the developing C57BL/6 mouse brain. Brain Res Dev Brain Res. 2002;133(2):115-26. PMID: 11882342.

21. Oliveira SA, Chuffa LG, Fioruci-Fontanelli BA, Lizarte Neto FS, Novais PC, Tirapelli LF, Oishi JC, Takase LF, Stefanini MA, Martinez M, Martinez FE. Apoptosis of Purkinje and granular cells of the cerebellum following chronic ethanol intake. Cerebellum. 2014;13(6):728-38. PMID: 25129034.

22. Martinez M, Sauce R, Oliveira SA, de Almeida Chuffa LG, Stefanini MA, Lizarte Neto FS, Takase LF, Tirapelli LF, Martinez FE. Ethanol intake-induced apoptosis in glial cells and axonal disorders in the cerebellar white matter of UChA rats (voluntary ethanol consumers). Tissue Cell. 2015;47(4):389-94. PMID: 26072102.

\section{Correspondence:}

Luís Fernando Tirapelli

Avenida Bandeirantes, 3900

14049-900 Ribeirão Preto - SP Brasil

lab.biomol.cirurgia@fmrp.usp.br

Received: May 21, 2016

Review: July 19, 2016

Accepted: Aug 18, 2016

Conflict of interest: none

Financial source: none

${ }^{1}$ Research performed at Molecular Biology Laboratory, Department of Surgery and Anatomy of Medical School of Ribeirão Preto, Universidade de São Paulo (USP), Brazil. 